\title{
Hydropower unit model identification using the field data
}

\author{
Jiafu Wei ${ }^{1}$, Xingran $\mathrm{Guo}^{2}$, Changli Wu ${ }^{1}$, Yuanchu Cheng ${ }^{2}$, Liangliang Qiao ${ }^{1}$ \\ Guili Zou ${ }^{1}$ \\ (1 State Grid Hunan Province Electric Power Corporation Electric Power Research Institute, \\ Changsha, Hunan 410007, China) \\ (2 Power and Mechanical Engineering, Wuhan University, Wuhan, Hubei 430072, China) \\ Xingran Guo:1466652565@qq.com
}

\section{Keywords: hydropower unit, ARMA, least square method, model identification}

Abstract: A model identification method of hydropower unit (HU) based on Auto-Regressive and Moving Average (ARMA) has been proposed. The ARMA model coefficients were obtained by least square method. Using the proposed method, the parameters $e_{y}, e$, and $e_{q h}$ of hydro turbine can be calculated. Experiment results show that the model identification simulations were consistent with the measured data of field tests.

\section{Introduction}

Many hydropower plants have been built in China, which is the main power supply of the whole power system. The mathematical model accuracy of HU directly affects the correct response to the power system $[1,2]$. Some scholars have studied the mathematical modeling of HU which includes ideal hydraulic turbine model, linear hydraulic turbine model based on transfer coefficient, the IEEE simplified nonlinear hydraulic turbine model, nonlinear hydraulic turbine model based on model synthetic characteristic curve, and the internal characteristics hydraulic turbine model [3, 4, 5]. The model parameters identification of HU is still one of the urgent problems to be solved [6, 7]. The main existing problems are as follows: (1) There are various types of hydraulic turbine and the characteristics are very different. (2) Because of the strong nonlinearity and wide operating range of the turbine, there is lack of effective method for model identification [8-9]. Literature [10-11] summarizes the modeling of prime mover and its regulation system, which is an important reference for the power system modeling and parameter testing.

In the field of parameter identification, the traditional identification methods are the least squares method and its improved form, as well as the maximum likelihood parameter identification and its improved form. But the above two methods are sensitive to the input signal types and noise, and the identification of nonlinear systems is limited. In recent years, with the development of genetic algorithms, evolutionary algorithms, ant colony optimization, particle swarm optimization and other biological heuristic algorithms, they have been applied to parameter identification [8]. The parameters setting of these algorithms are based on experience and the whole system modeling is complex. These methods are more active in the field of research, but have little application in engineering. The classical identification methods are still mainly used in engineering application [12-19].

In this paper, a model identification method based on ARMA and least square method is 
proposed to identify the model of the hydraulic turbine. The engineering application example is given at the end of this paper.

\section{Linear Mathematical Model of HU}

There are two typical models of the hydro turbine: the linear model and nonlinear model. The linear model is obtained in stable condition. As the model structure is simple and is easy to calculate, it is widely used in China. In the Power System Analysis Software Package (PSASP) and Power Administration Bonneville (BPA) simulation software, its mathematical model is easy to construct for simulation calculation.

The linear hydraulic turbine model can be described as

$$
G_{t}(s)=\frac{M_{\mathrm{t}}(s)}{Y(s)}=e_{y} \frac{1+e G_{h}(s)}{1-e_{q h} G_{h}(s)}
$$

Where $G_{h}(s), M_{t}(s)$ and $Y(s)$ are transfer function of diversion system, turbine torque and the turbine guide vane opening, respectively.

$e$ can be written as $e=e_{q y}{ }^{*} e_{h} / e_{y}-e_{q h}$, the four constants of hydro turbine $e_{y}, e_{h}, e_{q y}, e_{q h}$ are the partial derivatives of the torque and flow with respect to gate opening and head, respectively.

The rigid water hammer model that is employed in the paper can be described as

$$
G_{h}(s)=-\mathrm{T}_{\mathrm{w}} s
$$

Where $T_{w}$ denotes water flow inertia time constant.

Then the transfer function of the turbine and conduit system is

$$
G_{t}(s)=\frac{M_{\mathrm{t}}(s)}{Y(s)}=e_{y} \frac{1-e \mathrm{~T}_{\mathrm{w}} s}{1+e_{q h} \mathrm{~T}_{\mathrm{w}} s}
$$

Setting parameters $e_{y}=1.0, e_{h}=1.5, e_{q y}=1.0$ and $e_{q h}=0.5$, respectively, the equation (3) can be written as

$$
G_{t}(s)=\frac{1-\mathrm{T}_{\mathrm{w}} s}{1+0.5 \mathrm{~T}_{\mathrm{w}} s}
$$

Equation (4) is the ideal model of hydro turbine for the current parameter identification. The unit operating conditions can be reflected by $T_{w}$. In engineering, if all calculation parameters are calculated based on the design conditions, $T_{w}$ can be described as

$$
T_{W}=\frac{L Q_{r}}{g A H_{r}}
$$

Obviously, $T_{w}$ is a constant value and $e_{y}>1$.

In this paper, the ARMA model of hydro generator unit is set up based on equation (3) and the operation conditions are reflected by means of $e_{y}, e$, and $e_{q h}$.

By equation (3), the difference equation of hydro turbine can be written as

$$
m_{t}(t)=\frac{e_{q h} T_{w}}{e_{q h} T_{w}+D t} m_{t}(t-D t)+\frac{e_{y}\left(D t-e T_{w}\right)}{e_{q h} T_{w}+D t} y(t)+\frac{e_{y} e T_{w}}{e_{q h} T_{w}+D t} y(t-D t)
$$

Equation (6) can be rewritten as 


$$
m_{t}(k)=-a m_{t}(k-1)+b y(k)+c y(k-1)
$$

Where $\Delta t$ is the sampling time step.

Relevant parameters in equation (7) are obtained by the following formulas:

$$
a=-\frac{e_{q h} T_{w}}{e_{q h} T_{w}+D t} \quad b=\frac{e_{y}\left(D t-e T_{w}\right)}{e_{q h} T_{w}+D t} \quad c=\frac{e_{y} e T_{w}}{e_{q h} T_{w}+D t}
$$

Obviously, equation (7) has the same expression as ARMA regression model. When $y=0$, it is actually an auto regressive (AR) model. For the standard ARMA model, the input $y(k)$ is a white noise sequence of zero mean and the variance is Sigma. According to the above discussion, the ARMA model of hydraulic turbine is established.

\section{Model identification based on ARMA}

There are many methods for ARMA model parameter estimation. If the input sequence $\{y(k)\}$ and the output sequence $\left\{m_{t}(k)\right\}$ both can be measured, the ARMA parameters can be estimated with sufficient accuracy by the least square method. Based on equation (7), a linear equation group can be obtained by a series of values, which can be rewritten as follows

$$
\left[\begin{array}{l}
\mathrm{m}_{t}(1) \\
\mathrm{m}_{t}(2) \\
\mathrm{m}_{t}(3) \\
\mathrm{M} \\
\mathrm{m}_{t}(l)
\end{array}\right]=\left[\begin{array}{lll}
-\mathrm{m}_{t}(0) & \mathrm{y}(1) & \mathrm{y}(0) \\
-\mathrm{m}_{t}(1) & y(2) & y(1) \\
-\mathrm{m}_{t}(2) & y(3) & y(2) \\
\mathrm{M} & \mathrm{M} & \mathrm{M} \\
-\mathrm{m}_{t}(l-1) & y(l) & y(l-1)
\end{array}\right]\left[\begin{array}{l}
\mathrm{a} \\
b \\
c
\end{array}\right]+\left[\begin{array}{l}
\mathrm{v}(1) \\
v(2) \\
v(3) \\
\mathrm{M} \\
v(l)
\end{array}\right]
$$

Where $v(k)$ and $l$ denote the noise signal and data length, respectively.

$$
\text { If } \quad \mathrm{Z}=\left[m_{\mathrm{t}}(1) m_{\mathrm{t}}(2) m_{\mathrm{t}}(3) \mathrm{L} m_{\mathrm{t}}(l-1)\right]^{T} \quad, \quad H=\left[\begin{array}{lll}
-\mathrm{m}_{t}(0) & \mathrm{y}(1) & \mathrm{y}(0) \\
-\mathrm{m}_{t}(1) & y(2) & y(1) \\
-\mathrm{m}_{t}(2) & y(3) & y(2) \\
\mathrm{M} & \mathrm{M} & \mathrm{M} \\
-\mathrm{m}_{t}(l-1) & y(l) & y(l-1)
\end{array}\right]
$$

$\mathrm{X}=\left[\begin{array}{lll}\mathrm{a} & b & c\end{array}\right]^{T}, \mathrm{~V}=[\mathrm{v}(1) v(2) v(3) \mathrm{L} v(l-1)]^{T}$, then equation (9) can be rewritten as follows

$$
\mathrm{Z}=H X+V
$$

Define minimum error criterion function as

$$
\mathrm{J}=(Z-H X)^{T} *(Z-H X)
$$

When $X=X^{*}$, the first order partial derivative of the minimum error criterion function is $\left.\frac{\partial \mathrm{J}}{\partial \mathrm{X}}\right|_{X=X^{*}}=-2\left(Z-H X^{*}\right) H=0$.

$X^{*}$ can be written as follows

$$
X^{*}=\left(H^{T} H\right)^{-1} H^{T} Z
$$


Based on equation (7), the parameters $a, b$ and $c$ can be described as

$$
\begin{aligned}
& \left.\frac{\partial \mathrm{J}}{\partial \mathrm{a}}\right|_{a=a^{*}}=\sum_{k=1}^{l}\left[m_{t}(k)+a m_{t}(k-1)-b y(k)-c y(k-1)\right] * m_{t}(k-1)=0 \\
& \left.\frac{\partial \mathrm{J}}{\partial \mathrm{a}}\right|_{\mathrm{b}=b^{*}}=\sum_{k=1}^{l}\left[m_{t}(k)+a m_{t}(k-1)-b y(k)-c y(k-1)\right]^{*} y(k)=0 \\
& \left.\frac{\partial \mathrm{J}}{\partial \mathrm{a}}\right|_{\mathrm{c}=c^{*}}=\sum_{k=1}^{l}\left[m_{t}(k)+a m_{t}(k-1)-b y(k)-c y(k-1)\right] * y(k-1)=0
\end{aligned}
$$

Based on equations (13), (14) and (15), $a, b$ and $c$ can be obtained, respectively. Then the parameters $e_{y}, e$, and $e_{q h}$ can be calculated according to equations(8) when $T_{w}$ is known.

\section{Results and discussion}

In order to make the parameter identification of the hydraulic turbine model, the TPI software is developed according to the above method. The parameters of the hydraulic turbine regulating system can be easily identified by the software.

As shown in Figure 1, black line is guide vane opening curve, and red line is power curve of the generator from field test. Identified parameters by using the field test data are shown as follows

$$
e_{y}=1.494847, e=0.034538, e_{q h}=0.491029, T_{w}=1.886000
$$

The yellow curve is that the unit output power has been calculated according to the field test guide vane opening and identified model. And the results are in good agreement with the field test data as shown of the red curve. The correctness of the model identification results is described.

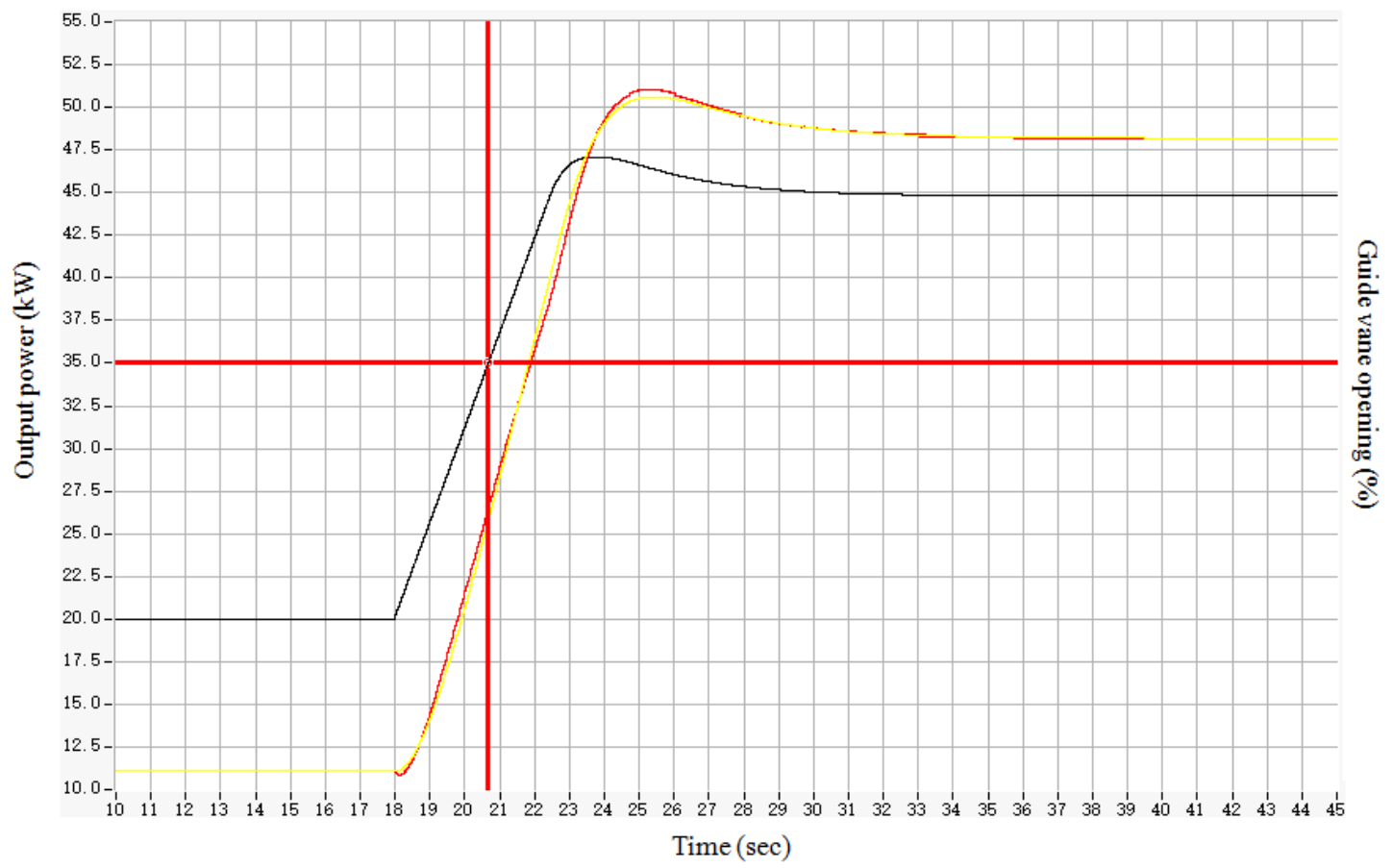

Figure 1 Model deification results of case one

\section{Conclusions}

ARMA regression model based on linear hydraulic turbine model is presented in this paper. The 
parameters relationship between ARMA and hydraulic turbine model is unique. $T_{w}$ is calculated under design conditions. The operating conditions of the unit are reflected by the changes of $e_{y}$, e and $e_{q h}$. The ARMA parameters are obtained by using the least square method according to the measured data. The results show that the hydraulic turbine model parameters can be obtained by using the presented method. Based on the identification results, the calculated output power can be in good agreement with the testing results. The validity and practicability of the method have been proved finally.

\section{References}

[1] S. R. Shakya and R. M. Shrestha, "Transport sector electrification in a hydropower resource rich developing country: energy security, environmental and climate change co-benefits," Energy for Sustainable Development, vol. 15, no. 2, pp. 147-159, 2011.

[2] Y.J. Li and X. L. Liu, "Power system transient simulation considering detailed models of hydraulic system," Power System Technology, vol.29, no.8, pp.61-64, 2015.

[3] N. Kishor, R. P. Saini, and S. P. Singh, "A review on hydropower plant models and control," Renewable and Sustainable Energy Reviews, vol. 11, no. 5, pp. 776-796, 2007.

[4] T. Bakka and H. R. Karimi, "Linear parameter-varying modeling and control of an offshore wind turbine with constrained information," IET Control Theory \& Applications, vol. 8, no. 1,pp. 22-29, 2014.

[5] C.Y. Liu, X.S. He and C.W. Li, etc. "Improved artificial fish swarm algorithm for parameter identification of hydroelectric turbine-conduit system," Electric Power Automation Equipment, vol.33,no.11,pp. 54-58,63, 2013.

[6] D. Kosterev, "Hydro turbine-governor model validation in pacificnorthwest," IEEE Transactions on Power Systems, vol.19,no.2,pp. 1144-1149,2004.

[7] F. Liu and Y.Y. Li, "Quantum clonal evolutionary algorithms," Acta Electronica Sinica, vol.31,no. 12A ,pp. 2066-2070,2003.

[8] C.Y. Qin, J.G. Zheng and J.J.Zhu, "Real-coded quantum-inspired evolutionary algorithm and its convergence," Control and Decision, vol.24, no.6, pp. 854-858, 2009.

[9] P. Pennacchi, S. Chatterton, and A. Vania, "Modeling of the dynamic response of a Francis turbine," Mechanical Systems and Signal Processing, vol. 29, pp. 107-119, 2012.

[10]Standardization Administration of the People's Republic of China. Mechanical Vibration-Evaluation of Machine Vibration by Measurements on Rotating Shafts-Part 5:Machine Sets in Hydraulic Power Generating and Pumping Plants. 2008, Beijing, China.

[11] Standardization Administration of the People's Republic of China. Code for field measurement of vibrations and pulsation in hydraulic machines (turbines storage pumps and pump-turbines). 2007, Beijing, China.

[12]N.Kishor, S. P. Singh, and A. S. Raghuvanshi, "Dynamic simulationsof hydro turbine and its state estimation based LQcontrol,'Energy Conversion andManagement, vol. 47, no. 18-19, pp. 3119-3137, 2006.

[13]M. Nässelqvist, R. Gustavsson and J. O. Aidanpää, “A methodology for protective vibration monitoring of hydropower units based on the mechanical properties," Journal of Dynamic Systems Measurement and Control, vol. 135, pp. 1-8, 2013.

[14]M. Nässelqvist , R. Gustavsson and J. O. Aidanpää, "Bearing load measurement in a 
hydropower unit using strain gauges installed inside pivot pin," Experimental Mechanics,vol.52, pp.361-369,2012.

[15] Y.S. Diao and H. Ren, "Structural damage early warning based on AR model and factor analysis," Journal of Vibration and Shock, vol. 33, pp.115-119, 2014.

[16]D.M Wang, L. Wang and G.M. Zhang, "Short-term wind speed forecast model for wind farms based on genetic BP neural network," Journal of Zhejiang University(Engineering Science), vol.46, pp.837-841,2012.

[17]G.D.G. Maria, C. Stefano, F. Antonio and M.C. Paolo, "Comparison between wind power predction models based on wavelet decomposition with least squares support vector machine (LS-SVM) and artificial neural network," Engergies, vol.7, pp.5251-5272,2014.

[18]M.A. Mathias, C. Mohamed and B. Alain, "Semisupervised learning using Bayesian interpretation: application to LS-SVM," IEEE Transactions on Neural Networks,vol. 22, pp.513-524, 2011.

[19]R. Gines, P. Hector, R. Ignacio and J.H. Luis, "A heuristic method for parameter selection in LS-SVM: application to time series prediction," International Journal of Forecasting, vol.27, pp.725-739, 2011. 